\title{
Dropwise Condensation on Nanostructured Surface
}

\author{
Jinliang Xu and Jian Xie \\ North China Electric Power University \\ Beijing, 102206, China \\ xjl@ncepu.edu.cn
}

Superhydrophobic nanostructure surface behaves the lotus effect to promote droplet detachment. Thus, it was once regarded as the promising surface to enhance dropwise condensation. Here, dropwise condensation on superhydrophobic nanostructure was investigated in following ways: (1) long-term dropwise condensation experiment, (2) effect of different droplet detachment modes on heat transfer, and (3) comprehensive dropwise condensation model. On fresh nano-grasses surface, two heat transfer regimes are identified: (1) higher heat transfer coefficients with droplet jumping, and (2) constant heat transfer coefficients with droplet rolling. Compared with smooth hydrophobic surface, the nanograss surface holds smaller droplet departure size but lower heat transfer coefficients. The one-week long term operation changes the jumping or rolling mode to sliding mode, yielding nano-grasses breakage and heat transfer deterioration.

Practically, the practical droplet detachment size is selected as the minimum value among those predicted by sliding, rolling and jumping. The criterion equations are established for onset of sliding and rolling independently. Coupling the two criterion equations yields the mode selection criterion between sliding and rolling, expressed in dimensionless parameters. It is found that the transition between rolling and sliding is only dependent on equilibrium contact angle $\square_{\mathrm{e}}$ : droplet slides for $\square_{\mathrm{e}}<126.3^{\circ}$; , droplet rolls for $\square_{\mathrm{e}}>147.0^{\circ}$. Thus, 147.0 is recommended as the contact angle boundary between hydrophobicity and super-hydrophobicity.

The newly presented mode selection criterion is incorporated into our dropwise condensation model, matching measured experiment data well. We conclude that the nanostructure surface introduces both positive and negative effects on dropwise condensation. The increased number of drop nucleation sites and decreased droplet detachment size have positive contribution on condensation, but the additional nano-porous thermal resistance is the negative effect to heat transfer. The overall condensation performance is the competition between positive effect and negative effect. The densely populated nanostructure is recommended to have better heat transfer performance. The heterogeneous surface with hydrophilic islands orderly populated on hydrophobic surface is suggested to sustain the long term operation without the structure failure.

Keywords: dropwise condensation; droplet detachment mode; long-term operation; comprehensive effect. 